\title{
Spatial decoherence near metallic surfaces
}

\author{
R. Fermani, ${ }^{*}$ S. Scheel, and P. L. Knight \\ Quantum Optics and Laser Science, Blackett Laboratory, Imperial College London, Prince Consort Road, \\ London SW7 2BW, United Kingdom
}

(Received 25 November 2005; published 8 March 2006)

\begin{abstract}
We present a first-principles derivation of spatial atomic-sublevel decoherence near dielectric and metallic surfaces. The theory is based on the electromagnetic-field quantization in absorbing dielectric media. We derive an expression for the time variation of the off-diagonal matrix element of the atomic density matrix for arbitrarily shaped substrates. For planar multilayered substrates we find that for small lateral separations of the atom's possible positions the spatial coherence decreases quadratically with the separation and inversely to the squared atom-surface distance.
\end{abstract}

DOI: 10.1103/PhysRevA.73.032902

PACS number(s): 34.50.Dy, 42.50.Nn

\section{INTRODUCTION}

New physical models for quantum information processing and quantum computation have been inspired recently by the experimental achievements in trapping and controlling ultracold neutral atoms [1-5]. The first experimental step to achieve a physical realization of a quantum computer with neutral atoms is to confine them in a definite region in space. The creation of microscopic guides and traps for neutral atoms moving close to surfaces is possible using nanofabricated structures that either carry currents or are based on permanently magnetized films. The idea at the base of atom chips has been put forward by Frisch and Segré [6] who realized that, when a homogeneous magnetic field ("bias field") is superimposed with the field created by a current flowing through a wire, the magnetic field vanishes on a line parallel to the current which can trap atoms in low-field seeking magnetic hyperfine sublevels.

One of the main requirements for a qubit is to be well isolated from a noisy environment to avoid decoherence, namely, the destruction of quantum superpositions due to the coupling of the atom cloud to the noisy chip environment. Although neutral atoms are considered good candidates as quantum systems since they have a small coupling to the environment, they still suffer from loss and decoherence. When atoms are trapped in atom chips, they are held close to the material surfaces. The small separation between the cold atom cloud and the macroscopic environment (usually at room temperature) raises the question of how strong the energy exchange will be, and which limit of atom confinement and height above the surface can ultimately be reached. Thermal fluctuations induce noise currents [7] in the materials the trap is made of, and fluctuations of the electromagnetic field are produced in the conducting body. Such fluctuating fields can be strong enough for an atom close to the surface to drive rf magnetic dipole transitions that flip its spin causing either its loss or decoherence of its quantum state.

In Refs. [8-14], atom loss due to thermally driven spin flips has been widely investigated and several experiments

\footnotetext{
*Electronic address: rachele.fermani@imperial.ac.uk
}

have confirmed the theoretical findings [15-17]. In this article we examine the influence of thermally induced spin flips on the coherence properties of atomic spatial superposition states. Such coherent superpositions can be thought of being created by tunneling through a shallow potential barrier in either a double-well potential or, more generally, an optical lattice structure [18]. The study of the latter has received much attention over the recent years for its potential application in quantum information processing (see, e.g., Refs. $[19,20])$. The derivation is carried out within the framework of the quantum electrodynamic theory for electromagnetic fields in dielectric media [21-28] which yields a firstprinciple description of the decoherence properties of spatial atomic superposition states.

This work is organized as follows. Section II introduces the basic notions of a quantized electromagnetic field in a dielectric medium. In Sec. III the density matrix of the atom is obtained in the presence of a fluctuating magnetic field and an expression for the spatial coherence is derived. We focus on a particular substrate geometry, a planarly multilayered structure, in Sec. IV for which the dyadic Green function is explicitly known.

\section{BASIC EQUATIONS}

It is well known that the quantum statistical properties of electromagnetic fields and their interactions with atomic systems can be strongly influenced by the presence of dielectric bodies. In the present context it is useful to formulate quantum electrodynamics (QED) on a dielectric-matter background [21-28]. The interaction between atomic systems and the electromagnetic field is typically treated in terms of the polarization and magnetization associated with the atomic charges. Let us restrict our attention to an isotropic but arbitrarily inhomogeneous medium whose polarization responds linearly and locally to the electric field. Causality and the dissipation-fluctuation theorem [29] then require that

$$
\mathbf{P}(\mathbf{r}, t)=\varepsilon_{0} \int_{0}^{\infty} d \tau \chi(\mathbf{r}, t) \mathbf{E}(\mathbf{r}, t-\tau)+\mathbf{P}_{N}(\mathbf{r}, t),
$$

where $\chi(\mathbf{r}, t)$ is the dielectric susceptibility (in the time domain) and $\mathbf{P}_{N}(\mathbf{r}, t)$ is the noise polarization associated with 
dissipative processes in the dielectric medium.

Using Maxwell's equations in Fourier space, we find that $\mathbf{E}(\mathbf{r}, \omega)$ obeys the Helmholtz equation

$$
\boldsymbol{\nabla} \times \boldsymbol{\nabla} \times \mathbf{E}(\mathbf{r}, \omega)-\frac{\omega^{2}}{c^{2}} \varepsilon(\mathbf{r}, \omega) \mathbf{E}(\mathbf{r}, \omega)=\omega^{2} \mu_{0} \mathbf{P}_{N}(\mathbf{r}, \omega),
$$

where the complex permittivity $\varepsilon(\mathbf{r}, \omega)=\varepsilon_{R}(\mathbf{r}, \omega)+i \varepsilon_{I}(\mathbf{r}, \omega)$ is defined by

$$
\varepsilon(\mathbf{r}, \omega)=1+\int_{0}^{\infty} d \tau e^{i \omega t} \chi(\mathbf{r}, \tau)
$$

The solution to Eq. (2) can then be written as

$$
\mathbf{E}(\mathbf{r}, \omega)=\omega^{2} \mu_{0} \int d^{3} \mathbf{r}^{\prime} \boldsymbol{G}\left(\mathbf{r}, \mathbf{r}^{\prime}, \omega\right) \mathbf{P}_{N}\left(\mathbf{r}^{\prime}, \omega\right)
$$

where the Green tensor $\boldsymbol{G}\left(\mathbf{r}, \mathbf{r}^{\prime}, \omega\right)$ is a second rank tensor that has to be determined from the partial differential equation

$$
\nabla \times \nabla \times \boldsymbol{G}\left(\mathbf{r}, \mathbf{r}^{\prime}, \omega\right)-\frac{\omega^{2}}{c^{2}} \varepsilon(\mathbf{r}, \omega) \boldsymbol{G}\left(\mathbf{r}, \mathbf{r}^{\prime}, \omega\right)=\delta\left(\mathbf{r}-\mathbf{r}^{\prime}\right) \boldsymbol{U}
$$

where $\boldsymbol{U}$ is the unit dyad. An important consequence of the differential equation (5) is the integral relation [25]

$$
\int d^{3} \mathbf{s} \frac{\omega^{2}}{c^{2}} \varepsilon_{I}(\mathbf{s}, \omega) \boldsymbol{G}(\mathbf{r}, \mathbf{s}, \omega) \boldsymbol{G}^{+}\left(\mathbf{r}^{\prime}, \mathbf{s}, \omega\right)=\operatorname{Im} \boldsymbol{G}\left(\mathbf{r}, \mathbf{r}^{\prime}, \omega\right) .
$$

Quantization of this theory then proceeds in the usual way [28]. First, a factor is split off from the (classical) noise polarization

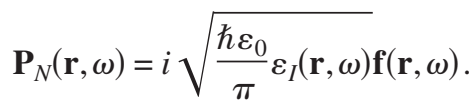

One then identifies the dynamical variables $\mathbf{f}(\mathbf{r}, \omega)$ as the fundamental $\delta$ correlated Gaussian random process and, upon quantization, replaces them by the operator-valued bosonic vector field $\hat{\mathbf{f}}(\mathbf{r}, \omega)$ satisfying the equal-time commutation relations $\left[\hat{\mathbf{f}}(\mathbf{r}, \omega), \hat{\mathbf{f}}^{\dagger}\left(\mathbf{r}^{\prime}, \omega^{\prime}\right)\right]=\delta\left(\mathbf{r}-\mathbf{r}^{\prime}\right) \delta\left(\omega-\omega^{\prime}\right) \boldsymbol{U}$. The Hamiltonian of the system composed of electromagnetic field and absorbing matter is

$$
\hat{H}_{F}=\int d^{3} \mathbf{r} \int_{0}^{\infty} d \omega \hbar \omega \hat{\mathbf{f}}^{\dagger}(\mathbf{r}, \omega) \hat{\mathbf{f}}(\mathbf{r}, \omega) .
$$

The electromagnetic field operators can now be obtained in the Schrödinger picture as

$$
\hat{\mathbf{E}}(\mathbf{r})=\int_{0}^{\infty} d \omega \hat{\mathbf{E}}(\mathbf{r}, \omega)+\text { H.c. }
$$

$$
\hat{\mathbf{E}}(\mathbf{r}, \omega)=i \sqrt{\frac{\hbar}{\pi \varepsilon_{0}}} \frac{\omega^{2}}{c^{2}} \int d^{3} \mathbf{r}^{\prime} \sqrt{\varepsilon_{I}\left(\mathbf{r}^{\prime}, \omega\right)} \boldsymbol{G}\left(\mathbf{r}, \mathbf{r}^{\prime}, \omega\right) \hat{\mathbf{f}}\left(\mathbf{r}^{\prime}, \omega\right)
$$

and, using Faraday's law,

$$
\hat{\mathbf{B}}(\mathbf{r}, \omega)=(i \omega)^{-1} \nabla \times \hat{\mathbf{E}}(\mathbf{r}, \omega) .
$$

An important feature of this theory is that it reproduces the correct form of the fluctuation-dissipation theorem. Let the system of electromagnetic field and absorbing matter be in thermal equilibrium at some temperature $T$. Then the thermal correlation function of the dynamical variables at temperature $T$ reads

$$
\left\langle\hat{\mathbf{f}}(\mathbf{r}, \omega) \hat{\mathbf{f}}^{\dagger}\left(\mathbf{r}^{\prime}, \omega^{\prime}\right)\right\rangle=\left(\bar{n}_{\mathrm{th}}+1\right) \delta\left(\mathbf{r}-\mathbf{r}^{\prime}\right) \delta\left(\omega-\omega^{\prime}\right) \boldsymbol{U},
$$

with the mean thermal photon number at frequency $\omega$

$$
\bar{n}_{\mathrm{th}}=\frac{1}{e^{\hbar \omega / k_{B} T}-1} .
$$

From Eqs. (10)-(12), together with Eq. (6), it follows that the thermal expectation value of an antinormally ordered product of magnetic field operators can be written as

$$
\begin{aligned}
\left\langle\hat{\mathbf{B}}(\mathbf{r}, \omega) \hat{\mathbf{B}}^{\dagger}\left(\mathbf{r}^{\prime}, \omega^{\prime}\right)\right\rangle= & \frac{\hbar \mu_{0}}{\pi} \operatorname{Im}\left[\vec{\nabla} \times \boldsymbol{G}\left(\mathbf{r}, \mathbf{r}^{\prime}, \omega\right) \times \overleftarrow{\nabla}\right] \\
& \times\left(\bar{n}_{\mathrm{th}}+1\right) \delta\left(\omega-\omega^{\prime}\right) .
\end{aligned}
$$

Such a quantization model provides a valid description of the electromagnetic field in absorbing dielectric materials. In fact, it has been shown in Refs. [24,25] that the fundamental equal-time commutation relations of QED are preserved. The electromagnetic field is expressed in terms of the classical Green tensor satisfying the Helmholtz equation (2), and the continuum of the bosonic field variables $\hat{\mathbf{f}}(\mathbf{r}, \omega)$. All the information about the dielectric matter is contained in the Green tensor via the permittivity $\varepsilon(\mathbf{r}, \omega)$. For metals at low frequencies, the permittivity can be approximated by the well-known Drude relation

$$
\varepsilon(\omega) \approx \frac{2 i c^{2}}{\omega^{2} \delta^{2}}
$$

with the skin depth $\delta$. Although such a relation is not strictly consistent with causality as it has recently been pointed out [30], it can be assumed to be valid in a restricted frequency interval.

At this stage it is necessary to notice the limitations of the quantization scheme presented above. Note that the form of the polarization (1) is valid only for strictly locally responding materials. That is to say, we assume that the elementary dipoles that give rise to the polarization are essentially fixed in space. Certainly, for metals which can alternatively be described by a conductivity, this is not true as charge carriers can move around freely for considerable distances. However, the locality assumption can be upheld in situations in which the mean free path length is much shorter than all the other length scales in the system under consideration. While this is certainly true for ordinary metals at room temperature and 
geometric length scales of several micrometers, we do expect corrections due to spatially nonlocal response (the anomalous skin effect) for metals or superconductors at very low temperatures as considered in Refs. $[13,14]$.

\section{SPATIAL DECOHERENCE}

Let us suppose we had an atom in one of two adjacent sites of an optical lattice. The tunneling interaction allows the atom's wave function to coherently spread over the neighboring site [31] where its state can be written in the occupation-number basis as

$$
|\psi(t=0)\rangle_{A}=\frac{1}{\sqrt{2}}(|1,0\rangle+|0,1\rangle) .
$$

We take the time at which the equal superposition has been established to be $t=0$ and assume for simplicity that no tunneling occurs at later times, at least not at time scales shorter than the decoherence time. This means that we imagine the tunneling interaction being frozen over a certain time period. This assumption is justified when considering proposals in which spatial atomic locations are used to encode quantum information.

Atoms that are held close to microstructured surfaces experience fluctuations of the electromagnetic field due to absorption in the substrate material. In the case of a magnetic trap the atom is subject to a constant magnetic field with strength $B_{0}$ in the center of the trap. The magnetic sublevels are split due to the Zeeman effect by the Larmor frequency $\omega_{L}=g_{S} \mu_{B} B_{0} / \hbar$. A subset of these magnetic sublevels feel an attractive potential towards regions of low magnetic field. In the experiment reported in Ref. [15] ${ }^{87} \mathrm{Rb}$ atoms are initially pumped into the hyperfine state $\left|F, m_{F}\right\rangle=|2,2\rangle$ in which they are trapped. However, due to absorption in the surface material and the resulting quantum fluctuations, fluctuating magnetic fields cause the atoms to evolve into states with lower magnetic quantum number $m_{F}$. In sufficiently tight magnetic traps, also atoms in the $\left|F, m_{F}\right\rangle=|2,1\rangle$ state are trapped. Spin flips to even lower magnetic sublevels cause the atoms to be expelled from the trap. In this case, spatial decoherence is no more a matter of interest. Hence, it is sufficient to treat the atomic system in a two-level approximation.

We focus on the Zeeman coupling of the atomic magnetic moment to a fluctuating field represented by the Hamiltonian

$$
\hat{H}_{Z}=-\hat{\mu} \cdot \hat{\mathbf{B}}\left(\mathbf{r}_{A}\right),
$$

where the operator of the magnetic induction is given by Eq. (11), together with Eqs. (9) and (10). The magnetic moment operator in Eq. (17) associated with a transition $|i\rangle \rightarrow|f\rangle$ can be written as $\hat{\boldsymbol{\mu}}=\boldsymbol{\mu}|i\rangle\langle f|+$ H.c. Since we assume the atom to be cooled into its electronic ground state, there is no contribution of the angular momentum. Furthermore, since the nuclear magnetic moment can be neglected because of the ratio of the electron mass to the mass of the nucleus (see the discussions in Refs. $[9,12]$ ), the magnetic moment vector is just proportional to the expectation value of the electronic spin operator

$$
\boldsymbol{\mu}=g_{S} \mu_{B}\langle i|\hat{\mathbf{S}}| f\rangle,
$$

where $\mu_{B}$ denotes the Bohr magneton, and $g_{S} \approx 2$ the electron's $g$ factor. Inserting Eq. (11) into Eq. (17), the Zeeman Hamiltonian can be written in the rotating-wave approximation as [12]

$$
\begin{aligned}
\hat{H}_{Z}= & -\mu_{B} g_{S}\left[\left\langle f\left|\hat{S}_{q}\right| i\right\rangle \hat{\xi}^{\dagger} \hat{B}_{q}\left(\mathbf{r}_{A}\right)+\text { H.c. }\right] \\
= & -\mu_{B} g_{S}\left[\left\langle f\left|\hat{S}_{q}\right| i\right\rangle \int_{0}^{\infty} d \omega \frac{\omega}{c^{2}} \sqrt{\frac{\hbar}{\varepsilon_{0} \pi}} \epsilon_{q p j} \partial_{p}\right. \\
& \left.\times \int d^{3} \mathbf{s} \sqrt{\varepsilon_{I}(\mathbf{s}, \omega)} G_{j i}\left(\mathbf{r}_{A}, \mathbf{s}, \omega\right) \hat{f}_{i}(\mathbf{s}, \omega) \hat{\xi}^{+}+\text {H.c. }\right],
\end{aligned}
$$

where $\hat{\xi}=|f\rangle\langle i|$ denotes the atomic spin lowering operator. Finally, the free atomic Hamiltonian can be written in the two-level approximation used above as

$$
\hat{H}_{A}=\hbar \omega_{A} \hat{\xi}_{z}=\frac{1}{2} \hbar \omega_{A}(|i\rangle\langle i|-| f\rangle\langle f|),
$$

where the $\hat{\xi}$ obey the commutation rules $\left[\hat{\xi}^{(\dagger)}, \hat{\xi}_{z}\right]=\mp \hat{\xi}^{(\dagger)}$.

In order to analyze how this magnetic noise influences the coherence of the state of our atom, we rewrite the initial atomic state as

$$
\left|\psi_{A}\right\rangle=\frac{1}{\sqrt{2}}\left(\left|i_{1}\right\rangle+\left|i_{2}\right\rangle\right)
$$

where the labels 1, 2 refer to the occupied site. Let us consider a system composed of the two-level atom and a fluctuating magnetic field initially in the vacuum state $|0\rangle$, so that the total state of the atom-field system reads

$$
\left|\psi_{A F}\right\rangle=\frac{1}{\sqrt{2}}\left(\left|i_{1}, 0\right\rangle+\left|i_{2}, 0\right\rangle\right)
$$

The Hamiltonian describing the evolution of the combined system is given by the sum of the three Hamiltonians $\hat{H}$ $=\hat{H}_{F}+\hat{H}_{A}+\hat{H}_{Z}$, where $\hat{H}_{F}, \hat{H}_{Z}$, and $\hat{H}_{A}$ are given by Eqs. (8), (20), and (19), respectively. The system wave function at a certain time $t$ can be written as [27]

$$
\begin{aligned}
\left|\psi_{A F}(t)\right\rangle= & C_{i_{1}}(t) e^{-i \omega_{A} t / 2}\left|i_{1}, 0\right\rangle+C_{i_{2}}(t) e^{-i \omega_{A} t / 2}\left|i_{2}, 0\right\rangle \\
& +\int d^{3} \mathbf{r} \int_{0}^{\infty} d \omega C_{f_{1}, m}(\mathbf{r}, \omega, t) e^{-i\left(\omega-\omega_{A} / 2\right) t}\left|f_{1}, 1_{m}(\mathbf{r}, \omega)\right\rangle \\
& +\int d^{3} \mathbf{r} \int_{0}^{\infty} d \omega C_{f_{2}, m}(\mathbf{r}, \omega, t) e^{-i\left(\omega-\omega_{A} / 2\right) t}\left|f_{2}, 1_{m}(\mathbf{r}, \omega)\right\rangle,
\end{aligned}
$$

where $|0\rangle$ and $\left|1_{m}(\mathbf{r}, \omega)\right\rangle$ denote the electromagnetic field vacuum and single-excitation states, respectively. The Schrödinger equation $i \hbar \partial_{t}\left|\psi_{A F}(t)\right\rangle=\hat{H}\left|\psi_{A F}(t)\right\rangle$ yields $\quad(a$ $=1,2$ ) 


$$
\begin{aligned}
\dot{C}_{i_{a}}(t)= & \frac{i \mu_{B} g_{S}}{c^{2} \sqrt{\pi \hbar \varepsilon_{0}}}\left\langle f\left|\hat{S}_{q}\right| i\right\rangle \int d^{3} \mathbf{r} \int_{0}^{\infty} d \omega \\
& \times \omega e^{-i\left(\omega-\omega_{A}\right) t} \sqrt{\varepsilon_{I}(\mathbf{r}, \omega)} \epsilon_{q p j} \partial_{p} G_{j m}\left(\mathbf{r}_{a}, \mathbf{r}, \omega\right) \\
& \times C_{f_{a}, m}(\mathbf{r}, \omega, t), \\
\dot{C}_{f_{a}, m}(\mathbf{r}, \omega, t)= & \frac{i \mu_{B} g_{S}}{c^{2} \sqrt{\pi \varepsilon_{0} \hbar}}\left\langle i\left|\hat{S}_{q}\right| f\right\rangle \omega e^{i\left(\omega-\omega_{A}\right) t} \\
& \times \sqrt{\varepsilon_{I}(\mathbf{r}, \omega)} \epsilon_{q p j} \partial_{p} G_{j m}^{*}\left(\mathbf{r}_{a}, \mathbf{r}, \omega\right) C_{i_{a}}(t) .
\end{aligned}
$$

We now substitute the result of formal integration of $C_{f_{a}, m}(\mathbf{r}, \omega, t)$ with the condition $C_{f_{a}, m}(\mathbf{r}, \omega, 0)=0$ into $\dot{C}_{i_{a}}(t)$, make use of the integral relation (6), and obtain

$$
\dot{C}_{i_{a}}(t)=\int_{0}^{t} d t^{\prime} K_{a}\left(t-t^{\prime}\right) C_{i_{a}}\left(t^{\prime}\right)
$$

where the integral kernel is

$$
\begin{aligned}
K_{a}\left(t-t^{\prime}\right)= & -\frac{\left(\mu_{B} g_{s}\right)^{2}}{c^{2} \pi \varepsilon_{0} \hbar}\left\langle f\left|\hat{S}_{q}\right| i\right\rangle\left\langle i\left|\hat{S}_{k}\right| f\right\rangle \\
& \times \int_{0}^{\infty} d \omega e^{-i\left(\omega-\omega_{A}\right)\left(t-t^{\prime}\right)} \\
& \times \operatorname{Im}\left[\vec{\nabla} \times \boldsymbol{G}\left(\mathbf{r}_{a}, \mathbf{r}_{a}, \omega\right) \times \overleftarrow{\nabla}\right]_{q k} .
\end{aligned}
$$

We integrate both sides of Eq. (26) over $t$, and changing the order of integrations on the right-hand side we derive

$$
C_{i_{a}}(t)-C_{i_{a}}(0)=\int_{0}^{t} d t^{\prime} \bar{K}_{a}\left(t-t^{\prime}\right) C_{i_{a}}\left(t^{\prime}\right)
$$

with

$$
\begin{aligned}
\bar{K}_{a}\left(t-t^{\prime}\right)= & \frac{\left(\mu_{B} g_{S}\right)^{2}}{c^{2} \pi \varepsilon_{0} \hbar}\left\langle f\left|\hat{S}_{q}\right| i\right\rangle\left\langle i\left|\hat{S}_{k}\right| f\right\rangle \\
& \times \int_{0}^{\infty} d \omega \frac{e^{-i\left(\omega-\omega_{A}\right)\left(t-t^{\prime}\right)}-1}{i\left(\omega-\omega_{A}\right)} \\
& \times \operatorname{Im}\left[\vec{\nabla} \times \boldsymbol{G}\left(\mathbf{r}_{a}, \mathbf{r}_{a}, \omega\right) \times \stackrel{\leftarrow}{\nabla}\right]_{q k}
\end{aligned}
$$

and the initial condition $C_{i_{a}}(0)=1$. When the Markov approximation applies, i.e., when in coarse grained description of the atomic motion memory effects are disregarded, we may let [32]

$$
\frac{\left(e^{-i\left(\omega-\omega_{A}\right)\left(t-t^{\prime}\right)}-1\right)}{i\left(\omega-\omega_{A}\right)} \rightarrow-\pi \delta\left(\omega-\omega_{A}\right)+i \mathcal{P} \frac{1}{\omega-\omega_{A}} .
$$

Defining the coefficients

$$
\begin{aligned}
& \Gamma_{a}=2\left(\frac{\left(\mu_{B} g_{S}\right)^{2}}{c^{2} \varepsilon_{0} \hbar}\right)\left\langle f\left|\hat{S}_{q}\right| i\right\rangle\left\langle i\left|\hat{S}_{k}\right| f\right\rangle \\
& \times \operatorname{Im}\left[\vec{\nabla} \times \boldsymbol{G}\left(\mathbf{r}_{a}, \mathbf{r}_{a}, \omega_{A}\right) \times \overleftarrow{\nabla}\right]_{q k}
\end{aligned}
$$

and

$$
\begin{aligned}
\delta \omega_{a}= & \left(\frac{\left(\mu_{B} g_{S}\right)^{2}}{c^{2} \pi \varepsilon_{0} \hbar}\right)\left\langle f\left|\hat{S}_{q}\right| i\right\rangle\left\langle i\left|\hat{S}_{k}\right| f\right\rangle \\
& \times \mathcal{P} \int_{0}^{\infty} d \omega \frac{\operatorname{Im}\left[\vec{\nabla} \times \boldsymbol{G}\left(\mathbf{r}_{a}, \mathbf{r}_{a}, \omega_{A}\right) \times \overleftarrow{\nabla}\right]_{q k}}{\omega-\omega_{A}},
\end{aligned}
$$

we can write $\bar{K}_{a}\left(t-t^{\prime}\right)=-\frac{1}{2} \Gamma_{a}+i \delta \omega_{a}$. We finally obtain for the time evolution of the coefficients $C_{i_{a}}(t)$

$$
C_{i_{a}}(t)=\exp \left[\left(-\frac{1}{2} \Gamma_{a}+i \delta \omega_{a}\right) t\right] .
$$

The coefficients $\Gamma_{a}$ and $\delta \omega_{a}$ defined in Eqs. (31) and (32) represent the spin flip rate and the line shift, respectively, and have been derived in a similar fashion in [12]. The spin flip lifetimes $1 / \Gamma_{a}$ have already been subject of major theoretical [8-14] and experimental [15-17] investigations which will not be repeated here. In what follows, we will assume that the line shift $\delta \omega_{a}$ caused by the interaction with the quantized electromagnetic field is negligible. This can be seen as follows. The Green function appearing in Eq. (4), as well as the Fourier transform of the permittivity in Eq. (1), plays the role of a response function and so it satisfies the KramersKronig relations for a complex-valued function $g(\omega)$ $=\operatorname{Re}[g(\omega)]+i \operatorname{Im}[g(\omega)][33]$,

$$
\begin{gathered}
\operatorname{Re}[g(\omega)]=\frac{1}{\pi} \mathcal{P} \int_{-\infty}^{\infty} d \omega^{\prime} \frac{\operatorname{Im}[g(\omega)]}{\omega^{\prime}-\omega}, \\
\operatorname{Im}[g(\omega)]=-\frac{1}{\pi} \mathcal{P} \int_{-\infty}^{\infty} d \omega^{\prime} \frac{\operatorname{Re}[g(\omega)]}{\omega^{\prime}-\omega} .
\end{gathered}
$$

The lower limit of the integral in Eq. (32) can be extended to $-\infty$ with little error as the integrand is peaked around $\omega_{A}$. Hence, Eq. (32) can be rewritten as

$$
\begin{aligned}
\delta \omega_{a}= & \left(\frac{\left(\mu_{B} g_{S}\right)^{2}}{c^{2} \varepsilon_{0} \hbar}\right)\left\langle f\left|\hat{S}_{q}\right| i\right\rangle\left\langle i\left|\hat{S}_{k}\right| f\right\rangle \\
& \times \operatorname{Re}\left[\vec{\nabla} \times \boldsymbol{G}\left(\mathbf{r}_{a}, \mathbf{r}_{a}, \omega_{A}\right) \times \overleftarrow{\nabla}\right]_{q k} .
\end{aligned}
$$

As we will see later, the line shift is of the same order of magnitude as the spin flip rate. For typical experimental realizations [9-17] this will be in the sub-Hz range. This means that $\delta \omega_{a}$ can be neglected as it is extremely small when compared to the spin flip transition frequency.

Now substituting Eq. (33) into the expression for $\dot{C}_{f_{a}, m}(\mathbf{r}, \omega, t)$, Eq. (25), we find the formal solution

$$
\begin{aligned}
C_{f_{a}, m}(\mathbf{r}, \omega, t)= & \frac{i \mu_{B} g_{S}}{c^{2} \sqrt{\pi \varepsilon_{0} \hbar}}\left\langle i\left|\hat{S}_{q}\right| f\right\rangle \omega \sqrt{\varepsilon_{I}(\mathbf{r}, \omega)} \epsilon_{q p j} \partial_{p} \\
& \times G_{j m}^{*}\left(\mathbf{r}_{a}, \mathbf{r}, \omega\right) \int_{0}^{t} d t^{\prime} e^{i\left(\omega-\omega_{A}\right) t^{\prime}} e^{-(1 / 2) \Gamma_{a} t^{\prime}} .
\end{aligned}
$$

In order to find how the off-diagonal elements of the density matrix decay, we trace the atomic density matrix over the field and obtain 


$$
\begin{aligned}
\varrho_{A}(t) & =\left\langle 0\left|\varrho_{A F}(t)\right| 0\right\rangle+\sum_{i} \int d^{3} \mathbf{r} \int_{0}^{\infty} d \omega\left\langle 1_{i}(\mathbf{r}, \omega)\left|\varrho_{A F}\right| 1_{i}(\mathbf{r}, \omega)\right\rangle \\
& =\frac{1}{2}\left(\begin{array}{cc}
\rho_{11}(t) & \rho_{12}(t) \\
\rho_{12}^{*}(t) & \rho_{22}(t)
\end{array}\right)
\end{aligned}
$$

where the matrix elements $\varrho_{i j}$ of the density matrix have to be calculated from

$$
\begin{aligned}
\varrho_{11}(t)= & \left|C_{i_{1}}(t)\right|^{2}+\sum_{i} \int d^{3} \mathbf{r} \int_{0}^{\infty} d \omega\left|C_{f_{1}, m}(\mathbf{r}, \omega, t)\right|^{2}, \\
\varrho_{22}(t)= & \left|C_{i_{2}}(t)\right|^{2}+\sum_{i} \int d^{3} \mathbf{r} \int_{0}^{\infty} d \omega\left|C_{f_{2}, m}(\mathbf{r}, \omega, t)\right|^{2}, \\
\varrho_{12}(t)= & C_{i_{1}}(t) C_{i_{2}}^{*}(t) \\
& +\sum_{i} \int d^{3} \mathbf{r} \int_{0}^{\infty} d \omega C_{f_{1}, m}(\mathbf{r}, \omega, t) C_{f_{2}, m}^{*}(\mathbf{r}, \omega, t) .
\end{aligned}
$$

First, it can be checked that the diagonal elements $\varrho_{11}(t)$ and $\varrho_{22}(t)$ are properly normalized to $\varrho_{11}(t)=\varrho_{22}(t)=1$ by inserting Eqs. (33) and (37) together with Eq. (6) into Eqs. (39) and (40), respectively. Thus, as a consistency check we find that $\operatorname{Tr}\left[\varrho_{A}\right]=1$. We can then calculate the off-diagonal elements of the density matrix as

$$
\begin{aligned}
\varrho_{12}(t)= & e^{-\Gamma_{12} t}+2\left(1-e^{-\Gamma_{12} t}\right) \frac{\left(\mu_{B} g_{S}\right)^{2}}{c^{2} \varepsilon_{0} \hbar}\left\langle i\left|\hat{S}_{q}\right| f\right\rangle \\
& \times\left\langle f\left|\hat{S}_{k}\right| i\right\rangle \frac{\operatorname{Im}\left[\overrightarrow{\boldsymbol{\nabla}} \times \boldsymbol{G}\left(\mathbf{r}_{2}, \mathbf{r}_{1}, \omega_{A}\right) \times \overleftarrow{\nabla}\right]_{k q}}{\Gamma_{12}},
\end{aligned}
$$

where $\Gamma_{12}=\left(\Gamma_{1}+\Gamma_{2}\right) / 2$ is the arithmetic mean of the spin flip rates, Eq. (31), at both sites. Note that the Hermiticity of the density matrix $\varrho_{A}(t)$ follows from the reciprocity theorem applied to the dyadic Green function which yields $\boldsymbol{G}\left(\mathbf{r}_{1}, \mathbf{r}_{2}, \omega_{A}\right)=\boldsymbol{G}^{T}\left(\mathbf{r}_{2}, \mathbf{r}_{1}, \omega_{A}\right)$.

Equation (42) constitutes the main result of our paper. It provides, via the Green function $\boldsymbol{G}\left(\mathbf{r}_{2}, \mathbf{r}_{1}, \omega_{A}\right)$, an elegant way to assess the loss of spatial coherence for arbitrarily shaped substrates. Recalling the expression for the fluctuation-dissipation theorem (14) it follows that Eq. (42) can be rewritten as

$$
\begin{aligned}
\varrho_{12}(t)= & e^{-\Gamma_{12} t}+\left(1-e^{-\Gamma_{12} t}\right) \\
& \times \frac{\left\langle i\left|\hat{S}_{q}\right| f\right\rangle\left\langle f\left|\hat{S}_{k}\right| i\right\rangle \int_{0}^{\infty} d \omega\left\langle\hat{B}_{k}\left(\mathbf{r}_{2}, \omega_{A}\right) \hat{B}_{q}^{\dagger}\left(\mathbf{r}_{1}, \omega\right)\right\rangle}{\left\langle i\left|\hat{S}_{q}\right| f\right\rangle\left\langle f\left|\hat{S}_{k}\right| i\right\rangle \int_{0}^{\infty} d \omega\left\langle\hat{B}_{k}\left(\mathbf{r}_{1}, \omega_{A}\right) \hat{B}_{q}^{\dagger}\left(\mathbf{r}_{1}, \omega\right)\right\rangle} \\
\equiv & e^{-\Gamma_{12} t}+\left(1-e^{-\Gamma_{12} t}\right) S\left(\mathbf{r}_{1}, \mathbf{r}_{2}, \omega_{A}\right)
\end{aligned}
$$

in terms of the magnetic cross-correlation tensor
$\left\langle\hat{\mathbf{B}}(\mathbf{r}, \omega) \hat{\mathbf{B}}^{\dagger}\left(\mathbf{r}^{\prime}, \omega^{\prime}\right)\right\rangle$. This means that the imaginary part of the (magnetic) Green function is proportional to the spatial coherence function of the fluctuating magnetic field [34-36].

Note that, although the calculations have been performed for surfaces held at zero temperature, the extension to finite temperatures is trivial. Indeed, it is seen from Eq. (14) that the spatial coherence functions as well as the spin-flip rates simply have to be multiplied by the factor $\left(\bar{n}_{\mathrm{th}}+1\right)$ to account for thermal fluctuations.

Equation (42) or, equivalently, Eq. (43), consists of two parts. The first is a (spatially local) exponential decay that describes the effect of the transition from the initial spin state $|i\rangle$ to the final spin state $|f\rangle$. The second term is a (spatially nonlocal) non-exponential term which is proportional to the spatial coherence function. It should be noted that, in a model in which more than a two-level transition is considered, after this time a transition to even lower-lying hyperfine spin states are likely. However, in our two-level approximation these flips are not taken into consideration.

\section{PLANAR MULTILAYER SUBSTRATES}

Up until now, the derivation of all formulas were valid for arbitrary substrate geometries. A particular geometric arrangement is fixed by defining the correct boundary conditions for the dyadic Green function $\boldsymbol{G}(\mathbf{r}, \mathbf{s}, \omega)$. In this section, we will concentrate on the simplest but experimentally important realization in terms of planar multilayer dielectrics. In what follows, we will focus on the spatially nonlocal term in Eq. (42) only. In particular, we notice that this is equivalent to taking the long-time limit of Eq. (42). Hence, for now we consider only

$$
\begin{aligned}
S\left(\mathbf{r}_{1}, \mathbf{r}_{2}, \omega_{A}\right)= & 2 \frac{\left(\mu_{B} g_{S}\right)^{2}}{c^{2} \varepsilon_{0} \hbar}\left\langle i\left|\hat{S}_{q}\right| f\right\rangle\left\langle f\left|\hat{S}_{k}\right| i\right\rangle \\
& \times \frac{\operatorname{Im}\left[\overrightarrow{\boldsymbol{\nabla}} \times \boldsymbol{G}\left(\mathbf{r}_{2}, \mathbf{r}_{1}, \omega_{A}\right) \times \overleftarrow{\nabla}\right]_{k q}}{\Gamma_{12}},
\end{aligned}
$$

which had previously been derived in connection with spatial decoherence of matter waves in Ref. [36]. Note that in a planar geometry in which the atom is held at a fixed distance to the material surface, the spin flip rates $\Gamma_{i}$ coincide due to translational invariance, i.e., $\Gamma_{12} \equiv \Gamma_{1}=\Gamma_{2}$. Note also that Eq. (44) is temperature independent.

Let us first consider a half-space filled with a dielectric or metal of dielectric permittivity $\varepsilon(\omega)$ (see the discussion in Sec. II). We evaluate the spin matrix elements for the transition from one hyperfine ground state to another by the basis states through the Clebsch-Gordon coefficients $\left|F, m_{F}\right\rangle$ $=\Sigma_{m_{S} m_{I}} C_{F m_{F}}^{m_{S} m_{I}}\left|m_{S}, m_{I}\right\rangle$. For the ${ }^{87} \mathrm{Rb}$ ground-state transition $|2,2\rangle \rightarrow|2,1\rangle$, the nonzero matrix elements are $\left|\left\langle i\left|\hat{S}_{y, z}\right| f\right\rangle\right|$ $=1 / 4$. The dyadic Green function for such a situation can be found in Refs. [24,37-39]. We have collected some of the formulas in Appendix A. Note that in the expressions for the components of the generalized reflection coefficient (A3) the common factor $e^{i k_{1 z}\left(z+z^{\prime}\right)} \equiv e^{2 i k_{1 z} d}$ can be approximated by $e^{-2 d\left|k_{\|}\right|}$because the transition wavelength $\lambda=c /(2 \pi \omega)$ is by 


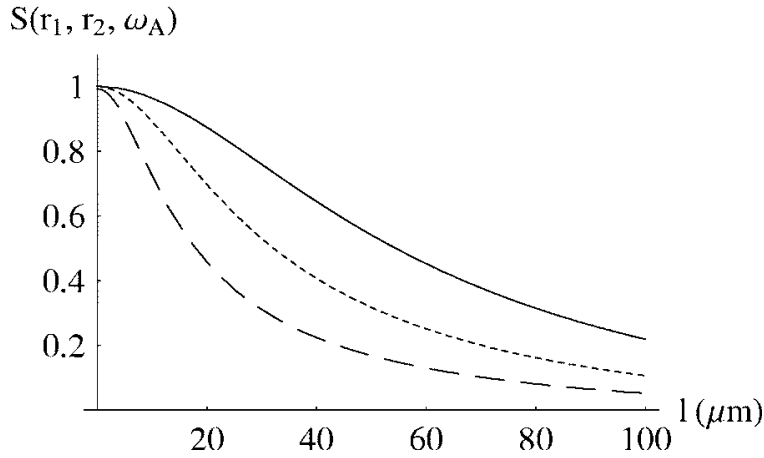

FIG. 1. Spatial coherence function of the fluctuating magnetic field $S\left(\mathbf{r}_{1}, \mathbf{r}_{2}, \omega_{A}\right)$, Eq. (44), as a function of the lateral separation $l$ in $\mu \mathrm{m}$ with the parameters $f=560 \mathrm{kHz}, \delta=110 \mu \mathrm{m}$ for three different distances from the surface: $d=20 \mu \mathrm{m}$ (solid line), $10 \mu \mathrm{m}$ (dotted line), and $5 \mu \mathrm{m}$ (dashed line).

far the biggest length scale in the system such that the approximation $k_{1 z}^{2} \approx-k_{\|}^{2}$ holds. Then, by going over to polar coordinates in the two-dimensional Fourier transform in Eq. (A2), $\quad \mathbf{k}_{\|}=\left(k_{x}, k_{y}\right) \mapsto(K \cos \varphi, K \sin \varphi)$, and $d^{2} \mathbf{k}_{\|} \mapsto K d K d \varphi$. We can thus write $\Gamma_{12}$ after integration over $\varphi$ as

$$
\Gamma_{12}=\frac{\left(\mu_{B} g_{S}\right)^{2}}{8 c^{2} \varepsilon_{0} \hbar} 3 \pi \int \frac{K^{2} d K}{(2 \pi)^{2}} \frac{e^{-2 K d}}{2} \operatorname{Im}\left[r_{12}^{\mathrm{TE}}\right]
$$

It is worth noting at this point that the line shift $\delta \omega_{a}$ in Eq. (36) can be computed as in Eq. (45) by replacing $\operatorname{Im}\left[r_{12}^{\mathrm{TE}}\right]$ with $\operatorname{Re}\left[r_{12}^{\mathrm{TE}}\right]$. Moreover, it is easily seen that both $\Gamma_{a}$ and $\delta \omega_{a}$ are of the same order.

Let us assume that an atom is located at a distance $d$ away from the planar interface which we describe by its skin depth $\delta$. In our example, we have chosen an aluminium substrate with $\delta=110 \mu \mathrm{m}$ and an atomic transition frequency as $f$ $=560 \mathrm{kHz}$. Furthermore, the atom can be in two distinct positions with a lateral separation $l$. In Fig. 1 we show the decay of the spatial coherence as measured by the function $S\left(\mathbf{r}_{1}, \mathbf{r}_{2}, \omega_{A}\right)$ for varying separation $l$ in $\mu \mathrm{m}$ for three different atom-surface distances $d$. As a function of separation, the decay of the spatial coherence starts off rather slowly. We attribute this behavior to the fact that for separations below the coherence length of the magnetic-field fluctuations the spin flip is driven coherently at both sites.

In order to investigate the small-separation limit in some more detail, we take a closer look at the Weyl expansion of the scattering Green tensor $\boldsymbol{R}^{(12)}\left(\mathbf{r}, \mathbf{r}^{\prime}, \omega\right)$, Eq. (A2), which is by far the dominant contribution compared with the freespace Green function. The separation $l$ is nothing but $l=\mid \varrho$ $-\boldsymbol{\varrho}^{\prime} \mid$ and serves as a parameter in the integral. Hence, we can expand the exponential $e^{i \mathbf{k}_{\| \cdot}\left(\varrho-\varrho^{\prime}\right)}$ in Eq. (A2) into powers of $l$ and evaluate each term seperately. The zeroth-order coefficient trivially leads to the spin flip rate $\Gamma_{12}$. The contribution from terms proportional to $l$ vanish identically due to the symmetry of the generalized reflection coefficients $R_{i j}^{(12)}$ with respect to the wave-vector components $\mathbf{k}_{\|}$in the $(x, y)$ plane. In fact, all odd powers of $l$ vanish because of that symmetry.
Hence, the lowest nonvanishing power is $l^{2}$. It is straightforward to find analytical expressions for the spatial coherence in that limit by converting the additional factor $K^{2}$ coming from the expansion of the exponential in Eq. (A2) into a parameter differentiation with respect to the atom-surface distance $d$. That is, we make the replacement $K^{2} \mapsto \frac{1}{4}\left(\partial^{2} / \partial d^{2}\right)$. In this way we find that

$$
S\left(\mathbf{r}_{1}, \mathbf{r}_{2}, \omega_{A}\right)=\frac{1}{\Gamma_{12}}\left(\Gamma_{12}-\frac{5 l^{2}}{96} \frac{\partial^{2}}{\partial d^{2}} \Gamma_{12}\right)+O\left(l^{4}\right) .
$$

In certain asymptotic regimes in which $\Gamma_{12}$ can be expressed as a monomial $\propto d^{-n}$ of the atom-surface distance $d$ (see, e.g., Refs. $[9,14,36])$, Eq. (46) can be rewritten in the form

$$
S\left(\mathbf{r}_{1}, \mathbf{r}_{2}, \omega_{A}\right)=1-\frac{5 n(n+1) l^{2}}{96 d^{2}}+O\left(l^{4}\right) .
$$

In addition to the planar half-space we consider the experimentally relevant situation in which a thin metallic layer of thickness $h$ has been brought onto a dielectric substrate. The generalized Fresnel coefficient for this three-layer system is given in Eq. (A5). In the limit of thick films $(\delta, h \gg d)$ the asymptotic behavior of the spin flip rate is $\Gamma_{12} \propto 1 / d[9,14]$ whereas for thin films $(\delta \gg d \gg h)$ we have $\Gamma_{12} \propto 1 / d^{2}$ $[14,36]$. Thus, we finally obtain the small- $l$ limit of Eq. (42) as

$$
\varrho_{12}(t)=1-\frac{5 \alpha l^{2}}{48 d^{2}}\left(1-e^{-\Gamma_{12} t}\right)+O\left(l^{4}\right),
$$

where $\alpha=1$ for thick films and $\alpha=3$ for thin films. It is interesting to note that the fall-off is three times faster for thin films than for thick films which we attribute to the fact that in thick films it is more likely to drive spin-flips coherently.

In order to see how the time scale is related to the expected lifetime we can expand the exponential in Eq. (48) for short times as

$$
\left|\varrho_{12}(t)-\varrho_{12}(0)\right| \cong \frac{5 \alpha l^{2}}{48 d^{2}}\left(\frac{t}{\tau}\right)+O\left(t^{2}\right)
$$

where $\varrho_{12}(0)=1$ and $\tau=\Gamma_{12}^{-1}$. The left-hand side in Eq. (49) can be thought as a proper measure of decoherence due to spin flips in terms of physical parameters such as the spinflip lifetime $\tau$, the separation $l$ and the distance from the surface $d$. This means that it is possible to maximize those experimental parameters while the decoherence rate is under control. Hence, Eq. (49) turns out to be particularly interesting from the quantum information point of view when a certain degree of spatial coherence has to be maintained.

For larger separations, however, it is difficult to find analytical approximations and one has to resort to numerical evaluations of the Fourier transform (A2). It is interesting to see at which separation $l_{1 / 2}$, as a function of the other length parameters in the system, the spatial coherence drops to half its initial value which could be taken as a measure of robustness. In Fig. 2 we show the dependence of $l_{1 / 2}$ on the thickness $h$ of the intermediate layer. In our calculations, we assumed a transition frequency of $f=560 \mathrm{kHz}$. We have plotted 


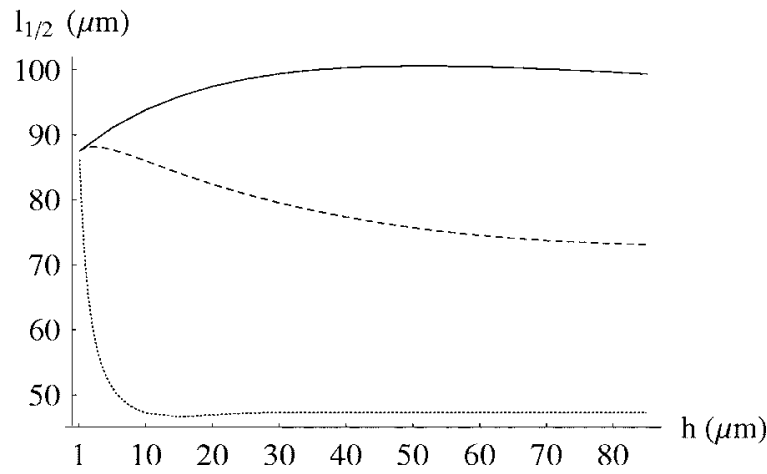

FIG. 2. Lateral separation $l_{1 / 2}$ after which spatial coherence has dropped to half its initial value as a function of the layer thickness $h$. The skin depth was varied from $\delta=100 \mu \mathrm{m}$ (solid line) to $\delta$ $=50 \mu \mathrm{m}$ (dashed line) and $\delta=10 \mu \mathrm{m}$ (dotted line). The atomsurface distance was $d=50 \mu \mathrm{m}$ and all other parameters as in Fig. 1.

$l_{1 / 2}$ for three different skin depths: $\delta=100 \mu \mathrm{m}$ (solid line, corresponding to a good conductor such as $\mathrm{Al}$ of $\mathrm{Cu}$ at room temperature), $\delta=50 \mu \mathrm{m}$ (dashed line), and $\delta=10 \mu \mathrm{m}$ (dotted line). Although the latter two skin depth values are not realistic for materials at room temperature, at cryogenic temperatures these values can be achieved. For example, just above its critical temperature of $T_{c}=9.2 \mathrm{~K}$, pure niobium shows a skin depth of only $\delta=15 \mu \mathrm{m}$ at $f \lesssim 1 \mathrm{MHz}$ [40].

In Fig. 2 it is clearly seen that for skin depths smaller than the atom-surface distance (dotted line), the robustness of spatial coherence drops dramatically with increasing substrate thickness $h$ until $h \sim \delta$. This can be understood when noting that by increasing the thickness of the intermediate layer one increases the number of fluctuating dipoles that can cause the spin flip. Any further increase beyond $h \sim \delta$ does not change much because fluctuations would not reach the substrate surface. Note also that the coherence length $l_{1 / 2}$ levels out roughly at the value of the skin depth, $l_{1 / 2} \sim \delta$.

For skin depths equal (dashed line in Fig. 2) or larger than the atom-surface distance (solid line) spatial coherence is robust over a wide range of substrate thicknesses $h$. Only for $h \gtrsim \delta$ does the coherence length decrease towards the atomsurface distance.

\section{CONCLUSIONS}

In summary, we have investigated loss of spatial coherence of atomic superpositions due to thermally driven spin flips. The consistent quantization of the electromagnetic field in absorbing dielectrics and metals allowed us to employ a first-principles approach to decoherence in this particularly simple physical system. The quantization scheme is based on the source-quantity representation of the electromagnetic field in terms of the dyadic Green function of the associated classical scattering problem and a bosonic vector field that serves as the dynamical variables of the theory. The Green function contains, via the dielectric permittivity, all information about the geometric arrangement and material properties of the substrate. Because the theory, starting already with Eq. (1), is strictly valid only for spatially locally responding ma- terials, we stress again that spatially nonlocal effects-which could be non-negligible for small skin depths (i.e., large conductivities) and small atom-surface distances-have not been considered.

The interaction dynamics between atomic spin and electromagnetic field has been described in the Schrödinger picture and the Markov approximation which led to the result for the time evolution of the off-diagonal matrix element (or coherence) $\varrho_{12}(t)$ of the single-particle density matrix (42). The spatially nonlocal part, Eq. (44), agrees with previously obtained results [36] for spatial decoherence of matter waves. It should be noted that both Eqs. (42) and (44) are valid for arbitrary geometrical arrangements of substrate materials.

For planarly multilayered substrates the dyadic Green function is explicitly known [24,37-39] and the main formulas presented in Appendix A. For small lateral separation $l$ of the atom's two possible positions we found that the spatial coherence decreases quadratically with $l$ and inversely proportional to the squared atom-surface distance $d$ [Eq. (48)]. For larger separations, a numerical study of a three-layer system showed that the coherence length $l_{1 / 2}$, defined to be the separation after which the coherence decays to half its initial value, converges for thick intermediate layers to roughly the atom-surface distance $d$.

We believe that these results are important for the design of microstructured devices in which spatial coherences are used to encode quantum information. In particular Eq. (49) shows how the decoherence rate depends on experimental parameters such as lifetime, lateral separation and atomsurface distance. They can be tuned in order to fall within a given tolerance rate for the degree of decoherence. Therefore, the theoretical results presented here may be useful in the physical realization of atomic traps where a certain degree of spatial coherence has to be maintained in order to be able to perform some kind of error correction.

\section{ACKNOWLEDGMENTS}

This work was financially supported by the UK Engineering and Physical Sciences Research Council (EPSRC) and the CONQUEST programme of the European commission.

\section{APPENDIX A: GREEN FUNCTION FOR PLANAR MULTILAYERS}

We briefly review the calculation of the Green function of planar multilayers as can be found in Refs. [24,37-39]. The dyadic Green function for the electric field scattering off a material interface can always be decomposed into

$$
\boldsymbol{G}\left(\mathbf{r}, \mathbf{r}^{\prime}, \omega\right)=\left\{\begin{array}{l}
\boldsymbol{G}^{(1)}\left(\mathbf{r}, \mathbf{r}^{\prime}, \omega\right)+\boldsymbol{R}^{(12)}\left(\mathbf{r}, \mathbf{r}^{\prime}, \omega\right) ; \mathbf{r}, \mathbf{r}^{\prime} \in \mathcal{V}_{1}, \\
\boldsymbol{T}^{(12)}\left(\mathbf{r}, \mathbf{r}^{\prime}, \omega\right) ; \mathbf{r} \in \mathcal{V}_{1}, \mathbf{r}^{\prime} \in \mathcal{V}_{2},
\end{array}\right.
$$

where $G^{(1)}\left(\mathbf{r}, \mathbf{r}^{\prime}, \omega\right)$ denotes the solution to the inhomogeneous Helmholtz equation with the source in region $\mathcal{V}_{1}$ which in our case is vacuum with $\varepsilon_{1}(\omega) \equiv 1$. The two (double-sided transverse) scattering parts $\boldsymbol{R}^{(12)}\left(\mathbf{r}, \mathbf{r}^{\prime}, \omega\right)$ and 
$\boldsymbol{T}^{(12)}\left(\mathbf{r}, \mathbf{r}^{\prime}, \omega\right)$ have to be introduced to satisfy the boundary conditions for the electromagnetic fields at the interface and describe the reflection and transmission parts of the total scattering Green function, respectively. These scattering Green functions satisfy the homogeneous Helmholtz equation. In our case, we only need to concentrate on the reflection part $\boldsymbol{R}^{(12)}\left(\mathbf{r}, \mathbf{r}^{\prime}, \omega\right)$.

The translational invariance in two spatial directions, say in the $(x, y)$ plane, allows one to write the Green function in terms of its Weyl expansion

$$
\boldsymbol{R}^{(12)}\left(\mathbf{r}, \mathbf{r}^{\prime}, \omega\right)=\int \frac{d^{2} \mathbf{k}_{\|}}{(2 \pi)^{2}} \boldsymbol{R}^{(12)}\left(\mathbf{k}_{\|}, \omega ; z, z^{\prime}\right) e^{i \mathbf{k}_{\|} \cdot\left(\boldsymbol{\rho}-\boldsymbol{\rho}^{\prime}\right)}
$$

$[\boldsymbol{\rho}=(x, y)]$, where $\mathbf{k}_{\|}=\left(k_{x}, k_{y}\right)$ is the wave vector in the $(x, y)$ plane. The matrix components of $\boldsymbol{R}^{(12)}\left(\mathbf{k}_{\|}, \omega ; z, z^{\prime}\right)$ can be read off from Ref. [24] as (here we omit the arguments to enhance readability)

$$
\begin{gathered}
R_{x x}^{(12)}=\frac{i}{2 k_{1 z}} e^{i k_{1 z}\left(z+z^{\prime}\right)}\left[-r_{12}^{\mathrm{TM}} \frac{k_{1 z}^{2} k_{x}^{2}}{k_{1}^{2} k_{\|}^{2}}+r_{12}^{\mathrm{TE}} \frac{k_{y}^{2}}{k_{\|}^{2}}\right], \\
R_{x y}^{(12)}=\frac{i}{2 k_{1 z}} e^{i k_{1 z}\left(z+z^{\prime}\right)}\left[-r_{12}^{\mathrm{TM}} \frac{k_{1 z}^{2} k_{x} k_{y}}{k_{1}^{2} k_{\|}^{2}}-r_{12}^{\mathrm{TE}} \frac{k_{x} k_{y}}{k_{\|}^{2}}\right], \\
R_{x z}^{(12)}=\frac{i}{2 k_{1 z}} e^{i k_{1 z}\left(z+z^{\prime}\right)}\left[r_{12}^{\mathrm{TM}} \frac{k_{1 z} k_{x}}{k_{1}^{2}}\right],
\end{gathered}
$$

$$
R_{z z}^{(12)}=\frac{i}{2 k_{1 z}} e^{i k_{1 z}\left(z+z^{\prime}\right)}\left[r_{12}^{\mathrm{TM}} \frac{k_{\|}^{2}}{k_{1}^{2}}\right],
$$

where $k_{i}^{2}=\left(\omega^{2} / c^{2}\right) \varepsilon_{i}(\omega)$ and $k_{i z}^{2}=k_{i}^{2}-k_{\|}^{2}$. The remaining matrix elements can be deduced from Eq. (A3) by replacement rules such as $R_{y y}^{(12)}=R_{x x}^{(12)}\left(k_{x} \leftrightarrow k_{y}\right)$ and the reciprocity condition $\boldsymbol{R}^{(12)}\left(\mathbf{r}, \mathbf{r}^{\prime}, \omega\right)=\boldsymbol{R}^{(12) T}\left(\mathbf{r}^{\prime}, \mathbf{r}, \omega\right)$ which yields $\boldsymbol{R}^{(12)}\left(\mathbf{k}_{\|}, \omega ; z, z^{\prime}\right)=\boldsymbol{R}^{(12) T}\left(-\mathbf{k}_{\|}, \omega ; z^{\prime}, z\right)$.

The functions $r_{12}^{\mathrm{TE}}$ and $r_{12}^{\mathrm{TM}}$ denote the usual Fresnel reflection coefficients for TE and TM waves, respectively, and are defined by

$$
r_{12}^{\mathrm{TE}}=\frac{k_{1 z}-k_{2 z}}{k_{1 z}+k_{2 z}}, \quad r_{12}^{\mathrm{TM}}=\frac{\varepsilon_{2}(\omega) k_{1 z}-\varepsilon_{1}(\omega) k_{2 z}}{\varepsilon_{2}(\omega) k_{1 z}+\varepsilon_{1}(\omega) k_{2 z}} .
$$

The Fresnel coefficients obey certain recursion relations that permit one to calculate the dyadic Green function for arbitrarily multilayered materials [37-39]. In particular, the generalized Fresnel coefficient for a three-layer geometry reads (for both TE and TM polarizations)

$$
\widetilde{r}_{12}=\frac{r_{12}+r_{23} e^{2 i k_{2 z} h}}{1-r_{21} r_{23} e^{2 i k_{2 z} h}},
$$

where $h$ is the thickness of the intermediate layer 2. This relation has been used in the numerical calculations throughout the paper.
[1] M. Greiner, I. Bloch, O. Mandel, T. W. Hänsch, and T. Esslinger, Phys. Rev. Lett. 87, 160405 (2001).

[2] M. Greiner, O. Mandel, T. Esslinger, T. W. Hänsch, and I. Bloch, Nature (London) 415, 39 (2002).

[3] J. Schmiedmayer, R. Folman, and T. Calarco, J. Mod. Opt. 49, 1375 (2002).

[4] D. Jaksch, H. J. Briegel, J. I. Cirac, C. W. Gardiner, and P. Zoller, Phys. Rev. Lett. 82, 1975 (1999).

[5] R. Ionicioiu and P. Zanardi, Phys. Rev. A 66, 050301(R) (2002).

[6] R. Frisch and E. Segré, Z. Phys. 75, 610 (1933).

[7] J. B. Johnson, Phys. Rev. 32, 97 (1928); H. Nyquist, ibid. 32, 110 (1928).

[8] T. Varpula and T. Poutanen, J. Appl. Phys. 55, 4015 (1984).

[9] C. Henkel, S. Pötting, and M. Wilkens, Appl. Phys. B 69, 379 (1999).

[10] R. Folman, P. Krüger, J. Schmiedmayer, J. Denschlag, and C. Henkel, Adv. At., Mol., Opt. Phys. 48, 263 (2002).

[11] C. Henkel, P. Krüger, R. Folman, and J. Schmiedmayer, Appl. Phys. B 76, 173 (2003).

[12] P. K. Rekdal, S. Scheel, P. L. Knight, and E. A. Hinds, Phys. Rev. A 70, 013811 (2004).

[13] C. Henkel, Eur. Phys. J. D 35, 59 (2005).

[14] S. Scheel, P. K. Rekdal, P. L. Knight, and E. A. Hinds, Phys. Rev. A 72, 042901 (2005).

[15] M. P. A. Jones, C. J. Vale, D. Sahagun, B. V. Hall, and E. A. Hinds, Phys. Rev. Lett. 91, 080401 (2003).
[16] Y. J. Lin, I. Teper, C. Chin, and V. Vuletić, Phys. Rev. Lett. 92, 050404 (2004).

[17] D. M. Harber, J. M. McGuirk, J. M. Obrecht, and E. A. Cornell, J. Low Temp. Phys. 133, 229 (2003).

[18] O. Mandel, M. Greiner, A. Widera, T. Rom, T. W. Hänsch, and I. Bloch, Nature (London) 425, 937 (2003).

[19] J. K. Pachos and P. L. Knight, Phys. Rev. Lett. 91, 107902 (2003).

[20] D. Jaksch, Contemp. Phys. 45, 367 (2004).

[21] W. Vogel, D.-G. Welsch, and S. Wallentowitz, Quantum Optics, An Introduction (Wiley-VCH, Weinheim, 2001).

[22] G. S. Agarwal, Phys. Rev. A 11, 230 (1975).

[23] T. Gruner and D.-G. Welsch, Phys. Rev. A 53, 1818 (1996).

[24] H. T. Dung, L. Knöll, and D.-G. Welsch, Phys. Rev. A 57, 3931 (1998).

[25] S. Scheel, L. Knöll, and D.-G. Welsch, Phys. Rev. A 58, 700 (1998).

[26] S. Scheel, L. Knöll, and D.-G. Welsch, Phys. Rev. A 60, 4094 (1999).

[27] H. T. Dung, L. Knöll, and D.-G. Welsch, Phys. Rev. A 62, 053804 (2000).

[28] L. Knöll, S. Scheel, and D.-G. Welsch, in Coherence and Statistics of Photons and Atoms, edited by J. Peřina (Wiley, New York, 2001).

[29] L. D. Landau and E. M. Lifshitz, Electrodynamics of Continuous Media (Pergamon, Oxford, 2000).

[30] A. Tip, Phys. Rev. E 69, 016610 (2004). 
[31] D. L. Haycock, P. M. Alsing, I. H. Deutsch, J. Grondalski, and P. S. Jessen, Phys. Rev. Lett. 85, 3365 (2000).

[32] S. M. Barnett and P. M. Radmore, Methods in Theoretical Quantum Optics (Oxford Science, Oxford, 1997).

[33] H. M. Nussenzveig, Causality and Dispersion Relations (Academic Press, New York, 1972).

[34] R. Carminati and J.-J. Greffet, Phys. Rev. Lett. 82, 1660 (1999).

[35] C. Henkel, K. Joulain, R. Carminati, and J.-J. Greffet, Opt. Commun. 185, 57 (2000).
[36] C. Henkel and S. Pötting, Appl. Phys. B 72, 73 (2001).

[37] M. S. Tomaš, Phys. Rev. A 51, 2545 (2000).

[38] W. C. Chew, Waves and Fields in Inhomogeneous Media (IEEE Press, New York, 1994).

[39] L. W. Li, P. S. Kooi, M. S. Leong, and T. S. Yeo, J. Electromagn. Waves Appl. 8, 663 (1994).

[40] S. Casalbuoni, E. A. Knabbe, J. Kötzler, L. Lilje, L. von Sawilski, P. Schmüser, and B. Steffen, Nucl. Instrum. Methods Phys. Res. A 538, 45 (2005). 